\title{
Effects of continuous intermedin infusion on blood pressure and hemodynamic function in spontaneously hypertensive rats
}

\author{
Ying Yuan ${ }^{1}$, Xi Wang ${ }^{2}$, Qiang Zeng ${ }^{3}$, Hong-Mei Wu ${ }^{2}$, Yong-Fen $\mathrm{Qi}^{4,5}$, Chao-Shu Tang ${ }^{4,5}$ \\ ${ }^{I}$ Human Centrifuge Medical Training Base of Institute of Aviation Medicine, Air Force, Beijing 100089, China \\ ${ }^{2}$ Department of Geriatrics Cardiology, Chinese PLA General Hospital, Beijing 100853, China \\ ${ }^{3}$ International Medical Center, Chinese PLA General Hospital, Beijing 100853, China \\ ${ }^{4}$ Department of Physiology, Health Science Center, Peking University, Beijing 100083, China \\ ${ }^{5}$ Institute of Cardiovascular Research, the First Hospital of Peking University, Beijing 100034, China
}

\begin{abstract}
Objective To examine the effects of exogenously administered intermedin (IMD, adrenomedullin-2) on arterial blood pressure, cardiac function and the cardiovascular IMD receptor system in spontaneously hypertensive rats (SHRs) as well as to investigate the associated mechanisms. Methods Thirteen week-old male rats were divided in Wistar Kyoto (WKY) group $(n=12)$, SHR group ( $n=12$ ), IMD group (SHRs infused with IMD 1-47 $500 \mathrm{ng} / \mathrm{kg}$ per hour, $n=12$ ), and ADM group (SHRs infused with adrenomedullin $500 \mathrm{ng} / \mathrm{kg}$ per hour, $n=12$ ). Results A two-week continuous administration of low dose IMD 1-47 via mini-osmotic pumps markedly reduced blood pressure, the maximal rates of increase and decrease of left-ventricle pressure development $\left(\mathrm{LV} \pm \mathrm{dp} / \mathrm{dt}_{\text {max }}\right)$, left ventricular systolic pressure and heart rate in SHRs. Furthermore, IMD also inhibited protein over-expression of cardiovascular IMD receptors, myocardial Receptor Activity-Modifying Proteins (RAMP1 and RAMP2), aortic RAMP1, RAMP2, RAMP3, and calcitonin receptor-like receptor (CRLR); suppressed up-regulation of aortic RAMP1, RAMP2, RAMP3 and CRLR gene expression; and markedly elevated the mRNA abundance of myocardial atrial natriuretic peptide (ANP) and myocardial brain natriuretic peptide (BNP). Additionally, IMD 1-47 administration in SHRs increased aortic cAMP concentration and reduced myocardial cAMP concentration. Conclusion These findings support the speculation that IMD, as a cardiovascular active peptide, is involved in blood pressure reduction and cardiac function amelioration during hypertension. The mechanism underlying this effect may involve IMD binding of a receptor complex formed by RAMPs and CRLR, and consequential regulation of cAMP levels and other cardiovascular active factors, such as ANP and BNP.
\end{abstract}

J Geriatr Cardiol 2012; 9: 17-27. doi: 10.3724/SP.J.1263.2012.00017

Keywords: Intermedin; Receptor activity-modifying proteins; Calcitonin receptor-like receptor; Mini-osmotic pumps; Hemodynamic parameters; Spontaneously hypertensive rats

\section{Introduction}

Intermedin (IMD) is a novel member of the calcitonin/ calcitonin gene-related peptide (CGRP) superfamily, comprising calcitonin, CGRP, adrenomedullin (ADM) and amylin. ${ }^{[1,2]}$ Takei et al. ${ }^{[3]}$ identified a novel member of the CGRP family, ADM2, in mammals. However, the amino acid and nucleotide sequence are nearly identical between human prepro-ADM2 and prepro-IMD, and ADM2 and IMD were found to be the same peptide. The human IMD

Correspondence to: Qiang Zeng, $\mathrm{PhD}$, International Medical Center, Chinese PLA General Hospital, Beijing 100853, China. E-mail: zq301@ hotmail.com

Telephone: +86-10-68295751

Fax: $+86-10-68295928$

Received: December 15, 2011

Revised: February 12, 2012

Accepted: February 19, 2012

Published online: March 28, 2012 gene encodes a preproprotein of 148 residues that is proteolyticly cleaved to yield two active fragments, a 47-amino acid peptide called intermedin 1-47 (IMD 1-47) and a 40-amino acid peptide called intermedin 8-47 (IMD 8-47). ${ }^{[4]}$ According to amino acid sequence analysis, the $93^{\text {rd }}$ and the $94^{\text {th }}$ amino acid residues of prepro-IMD are a pair of arginines, through which prepro-IMD can be degraded into prepro-AMD ${ }_{95-147}$, namely, $\mathrm{IMD}_{1-53}{ }^{[5]}$

Members of the CGRP family exert their effects through a calcitonin receptor-like receptor (CRLR) in the presence of a series of unique receptor activity modifying proteins (RAMPs, including RAMP1, RAMP2 and RAMP3). Combinations of different RAMPs and CRLR constitute receptors with selective ligand affinities. RAMP1-associated CRLR exhibits CGRP selectivity, while RAMP2-associated CRLR shows a preference for ADM. However, IMD elicits its biological function non-selectively through CRLR/RAMP1, 
CRLR/RAMP2 or CRLR/RAMP3, ${ }^{[1]}$ which is indicative of high desensitization resistance with stronger and more extensive biological effects. ADM and IMD are both considered potent vasodilators due to their ability to decrease arterial blood pressure, as well as inhibit urine flow, reduce food intake and suppress gastric activity when peripherally administered. ${ }^{[1,3,6-9]}$

Our previous studies showed that spontaneously hypertensive rats (SHRs) have a significantly higher arterial blood pressure, the maximal rates of increase and decrease of left-ventricle pressure development $\left(\mathrm{LV} \pm \mathrm{dp} / \mathrm{dt}_{\max }\right)$, left ventricular systolic pressure (LVSP) and heart weight/body weight (HW/BW) ratio than measured for the Wistar Kyoto (WKY) rats. Furthermore, SHRs exhibit elevated immunoreactive IMD levels in cardiovascular tissue and plasma, which are higher than the corresponding ADM concentrations. Both gene and protein expression of IMD and its receptors are up-regulated in the cardiovascular tissue of SHRs. The augmented reactivity of IMD and its receptor system boosts the anti-vasoconstriction activity of IMD on induced vasoconstriction during hypertension. This allows IMD's normal effects of promoting vasodilation and cardiac function amelioration to occur, which consequently prevents excessive blood pressure and guarantees normal myocardial perfusion. Thus, endogenous IMD has been speculated to exert a cardioprotective effect in the pathogenesis of hypertension. ${ }^{[10]}$

Intravenous administration of IMD in SHRs has been reported to decrease blood pressure and increase heart rate in a dose-dependent manner. ${ }^{[1,11]}$ The therapeutic response, however, varies with the infusion region and concentration. For example, intracerebroventricular injection of IMD 1-47 in Sprague Dawley rats elevates average arterial blood pressure and heart rate,$^{[12]}$ while intravenous injection of IMD 1-47 $(1.0 \mathrm{nmol} / \mathrm{kg})$ decreases the average arterial blood pressure and increases the heart rate. ${ }^{[3,12]}$ Furthermore, intravenously administered IMD at a low dose $(1.0 \mathrm{nmol} / \mathrm{kg})$ evokes a hypotensive effect comparable to that of CGRP, but weaker than that produced by $\mathrm{ADM}^{[12]}$ In contrast, increasing IMD 1-47 (3.0 nmol $/ \mathrm{kg}$ and $5.0 \mathrm{nmol} / \mathrm{kg})$ elicits a greater response than an equivalent dose of $\mathrm{ADM}^{[6,13]}$ Currently, hypertension is preferably treated with low dose medications in a slow consistent release formulation. This regimen can be conveniently simulated by the use of mini-osmotic pumps, which also allow for the avoidance of systemic side effects caused by regional drug administration compared to the previously employed intravenous administration.
IMD and its receptor system play pivotal roles in stabilizing blood circulation. However, their pathophysiology influences on cardiovascular diseases are still unclear. There is no report on the effect of IMD infusion via mini-osmotic pump on arterial blood pressure, cardiac function and the expression of the cardiovascular IMD receptor system in SHRs. Our study evaluated the therapeutic effect of continuous low dose IMD infusion on hypertension compared to a known antihypertensive agent from the same peptide family, ADM. We expected the results would shed light on the pathophysiology function of IMD and its receptor during hypertension and provide new insights into hypertension treatment.

\section{Methods}

\subsection{Animals and reagents}

All animal care and experimental protocols described in this study were in compliance with the Animal Management Rule of China (Ministry of Health, China, document No. 55, 2001) and the Animal Care Committee of the First Hospital, Peking University. Briefly, 13-week-old male SHRs weighing $300 \pm 20 \mathrm{~g}(n=36)$ and age/weight-matched healthy male WKY rats $(n=12)$ were maintained under normal conditions (temperature at $22 \sim 24^{\circ} \mathrm{C}$, humidity at $40 \% \sim 60 \%$ ) with free access to water and rat chow. ADM and IMD were purchased from the Phoenix Pharmaceutical Inc. (Belmont, CA, USA). The cAMP RIA kits were obtained from NEN Life Sci. Products Inc. (Boston, MA, USA). Antibodies specific for CRLR (sc-18007), RAMP1 (sc-11379), RAMP2 (sc11380) and RAMP3 (sc-11381) were purchased from Santa Cruz Biotechnology (Santa Cruz, Heidelberg, Germany). The anti $\beta$-actin antibody (sc-1616) and rabbit anti-sheep $\mathrm{IgG}$ and sheep anti-rabbit $\operatorname{IgG}$ secondary antibodies were all purchased from Friendship Biotechnology Co., Ltd. (Beijing, China). ImBlotter-NC nitrocellulose membrane, electrochemiluminescence (ECL) scintillation cocktail and the Trizol reagent were purchased from Beijing Applygen Technologies Corp. (Beijing, China). dNTP was purchased from Clontech (Palo Alto, CA, USA). M-MuLV reverse transcriptase, Taq DNA polymerase, and oligo (dT)15 primers were purchased from Promega (Madison, WI, USA). All other reagents were of analytical grade. All seven pairs of PCR primers were synthesized by Augct Technologies Corp. (Beijing, China) (Table 1). 
Table 1. Oligonucleotides used for the amplification.

\begin{tabular}{|c|c|c|c|c|c|}
\hline Rat Target & Sequence & & Size $^{\mathrm{a}}(\mathrm{bp})$ & $\operatorname{AnnT}^{\mathrm{b}}\left({ }^{\circ} \mathrm{C}\right)$ & Cycles \\
\hline \multirow[t]{2}{*}{ CRLR } & Sense & 5'-CAACTGCTGGATCAGCTCAG-3' & 249 & 60 & 35 \\
\hline & Antisense & 5'-CATCGCTGATTGTTGACACC-3' & & & \\
\hline \multirow[t]{2}{*}{ RAMP1 } & Sense & 5'-CAATCCGGAAGTGGACAAGTT-3' & 197 & 60 & 31 \\
\hline & Antisense & 5'-GACACCTACACGATGCCCTCT-3' & & & \\
\hline \multirow[t]{2}{*}{ RAMP2 } & Sense & 5'-CAAGGACTGGTGCAACTGGAC-3' & 257 & 60 & 31 \\
\hline & Antisense & 5'-ACACCACAAGCGTAACGAGGA-3' & & & \\
\hline \multirow[t]{2}{*}{ RAMP3 } & Sense & 5'ACCTGTCGGAGTTCATCGTGT-3' & 225 & 58 & 33 \\
\hline & Antisense & 5'-TAGCCACGGTCAACAAGACTG-3' & & & \\
\hline \multirow[t]{2}{*}{$\beta$-actin } & Sense & 5'-TCCTGGGTATGGAATCCTGTG-3' & 291 & 58 & 25 \\
\hline & Antisense & 5'-CTCAGGAGGAGCAATGATCTTG-3' & & & \\
\hline \multirow[t]{2}{*}{ ANP } & Sense & 5'-TCGAGCAGATCGCAAAAGATC-3' & 150 & 60 & 25 \\
\hline & Antisense & 5'-CACACTAAACCACTCATCTAC-3' & & & \\
\hline \multirow[t]{2}{*}{ BNP } & Sense & 5'-TCTGCTCCTGCTTTTCCTTA-3' & 256 & 60 & 26 \\
\hline & Antisense & 5'-GAACTATGTGCCATCTTGGA-3' & & & \\
\hline
\end{tabular}

${ }^{a}$ Expected sizes of the amplicons; ${ }^{\mathrm{b}} \mathrm{AnnT}$ : annealing temperature used for the PCR reaction. CRLR: Calcitonin receptor-like receptor; RAMP: Receptor activity-modifying proteins; ANP: atrial natriuretic peptide; BNP: brain natriuretic peptide.

\subsection{Continuous infusion of IMD/ADM}

SHRs or WKYs were continuously infused with IMD 1-47 or ADM for 14 days at a dose of $500 \mathrm{ng} / \mathrm{kg}$ per hour using mini-osmotic pumps (Model 2002, Alza Co., Durect, Cupertimo, California) following the manufacture's protocol. Briefly, male SHRs $(300 \pm 20 \mathrm{~g}, 13$ week, $n=36)$ were randomly divided into three groups: the IMD group (SHRs infused with IMD 1-47), the ADM group (SHRs infused with $\mathrm{ADM})$ and the SHR group. Male WKYs $(300 \pm 20 \mathrm{~g}$, 13 week, $n=12$ ) were utilized as control (WKY group). The mini-osmotic pumps were pre-incubated in sterile saline at $37^{\circ} \mathrm{C}$ for $12 \mathrm{~h}$ and filled with $100 \mu \mathrm{L}$ of saline solution containing IMD 1-47 or ADM. After anesthetization with sodium pentobarbital $(40 \mathrm{mg} / 100 \mathrm{~g})$, each rat was implanted with a pump subcutaneously through an approximately one centimeter incision on the back. The WKY and SHR groups were infused with only $100 \mu \mathrm{L}$ of saline solution using the same technique.

\subsection{Measurement of hemodynamic parameters in rats in vivo}

Blood pressure and cardiac function were measured using the BL-420E Biological System (Tai-Meng Biotechnological Company, Ltd., Chengdu, China). Rats were anesthetized with sodium pentobarbital $(40 \mathrm{mg} / 100 \mathrm{~g})$. A $0.7 \mathrm{~cm}$ neck incision was made to expose the right common carotid artery and a catheter was inserted approximately $1.5 \mathrm{~cm}$ towards the heart. Arterial blood pressure was monitored for $30 \mathrm{~min}$, and then the catheter was inserted into the ventricular chamber to record the intraventricular pressure for $30 \mathrm{~min}$. Hemodynamic indices monitored included systolic blood pressure (SBP), diastolic blood pressure (DBP), LVSP, left ventricular diastolic pressure (LVDP), LV \pm $\mathrm{dp} / \mathrm{dt}_{\max }$ and heart rate (HR). Rats were sacrificed by exsanguination from the abdominal aorta after measurement. One rat died following the hemodynamic measurements in each group. Eleven cases in each group were left for the following tests.

\section{4 mRNA levels of CRLR, RAMP1, RAMP2, RAMP3, ANP and BNP}

Total RNA was isolated from the myocardium and aorta using the TRIzol reagent (Invitrogen), followed by reverse transcription into cDNA using M-MuLV reverse transcriptase and the Oligo (dT) 15 primer. The total volume of CRLR mRNA PCR measurement was $10 \mu \mathrm{L}$, including $1 \mu \mathrm{L}$ of cDNA, $0.5 \mu \mathrm{L}$ of $5 \mathrm{pmol} / \mathrm{L}$ CRLR sense and anti-sense primers, $0.5 \mu \mathrm{L}$ of $2.5 \mathrm{mmol} / \mathrm{L} \mathrm{dNTP,} 1 \mu \mathrm{L}$ of $10 \times$ PCR buffer containing $15 \mathrm{mmol} / \mathrm{L} \mathrm{MgCl}_{2}$, and TaqDNA polymerase $0.5 \mathrm{IU}$. The thermocycler program involved denaturation at $94^{\circ} \mathrm{C}$ for $4 \mathrm{~min}, 94^{\circ} \mathrm{C}$ for $30 \mathrm{~s}, 60^{\circ} \mathrm{C}$ for $45 \mathrm{~s}, 72^{\circ} \mathrm{C}$ for $35 \mathrm{~s}$ for 33 cycles, then $72^{\circ} \mathrm{C}$ for $10 \mathrm{~min}$. PCR product $(5 \mu \mathrm{L})$ was separated by $1.2 \%$ agarose gel, stained with ethidium bromide (EB) and detected at 249 bp using densitometry. A similar technique was used to determine the mRNA levels of $\beta$-actin. Specific primers for $\beta$-actin (291 bp) were used, followed by thermocycling at $94^{\circ} \mathrm{C}$ for $30 \mathrm{~s}, 58^{\circ} \mathrm{C}$ for $45 \mathrm{~s}$ and $72^{\circ} \mathrm{C}$ for $35 \mathrm{~s}$ for 25 cycles. The ratio of CRLR mRNA to $\beta$-actin mRNA was used to assess the relative CRLR mRNA content. Similar approaches were used to determine the mRNA levels of RAMP1, RAMP2, RAMP3, ANP and BNP (Table 1). Data were obtained from three independent experiments. 


\subsection{Protein extraction and western blot analysis for CRLR and RAMP 1-3}

Protein extracts were prepared from the myocardium and aorta. Briefly, tissues were lysed and homogenized in a lysis buffer containing $20 \mathrm{mmol} / \mathrm{L}$ Tris- $\mathrm{HCl}$, pH 7.4 and $1 \mathrm{mmol} / \mathrm{L}$ EDTA. The samples were centrifuged at $1600 \mathrm{~g}$ for $10 \mathrm{~min}$ at $4^{\circ} \mathrm{C}$. Protein concentration was determined using the Bradford assay. Samples containing equal amount of proteins $(100 \mu \mathrm{g})$ were separated on SDS-PAGE gels and transferred to nitrocellulose membranes. The membranes were blocked for one hour with 5\% skim milk in Tris-buffered saline Tween-20 (TBS-T) containing $20 \mathrm{mmol} / \mathrm{L}$ Tris- $\mathrm{HCl}$, $\mathrm{pH} 7.5,0.05 \%$ Tween 20 , and $0.6 \% \mathrm{NaCl}$, followed by overnight incubation at $4^{\circ} \mathrm{C}$ with the appropriate primary antibodies in $0.05 \% \sim 0.1 \%$ TBS-T. Possible antibody choices were goat anti-rat CRLR (1: 400), goat anti-rat $\beta$-actin (1: 1000) and rabbit anti-rat RAMPs (1: 500 for RAMP1; 1 : 400 for RAMP2 and RAMP3) antibodies. The membranes were then incubated with horseradish peroxidase- conjugated secondary antibodies (i.e., anti-goat or anti-rabbit antibodies; 1 : 2000) at room temperature for one hour and then immediately visualized on a phosphor imager after addition of the ECL substrate. The band density was determined using NIH Image software after immunoblotting. The CRLR or RAMP contents were normalized using $\beta$-actin.

\subsection{Radioimmunoassay for cAMP content}

After in vivo and in vitro experiments, an adequate amount of the left ventricle of the myocardium and thoracic aorta were immediately immersed in $1 \mathrm{~mol} / \mathrm{L} \mathrm{HCl}$ and were boiled for $5 \mathrm{~min}$. Tissue homogenates were centrifuged at $2500 \mathrm{~g}$ for $10 \mathrm{~min}$ at $4^{\circ} \mathrm{C}$. The supernatant was collected, and the protein content was determined using the Bradford method. The samples were stored at $-70^{\circ} \mathrm{C}$ until assayed for cAMP content. Tissue cAMP content was measured by a highly sensitive ${ }^{125}$ I-radioimmunoassay kit, according to the manufacturer's instruction. The lower detection limit was 4 fmol per tube. The values were normalized to protein content.

\subsection{Statistical analysis}

GraphPad Prism 5.0 software was used for data analysis. All data were expressed as means $\pm \mathrm{SE}$ and analyzed via the Student's $t$-test for differences between groups. One-way analysis of variance (ANOVA) was performed to compare more than two groups with the Student-Newman-Keuls test used to assess differences between groups. Results with $P<0.05$ was considered statistically significant.

\section{Results}

\subsection{IMD improves hypertension and cardiac function in SHRs}

The SBP, DBP, $L V \pm d p / d_{\max }$ and HW/BW of the SHRs were all prominently higher than those of the WKY rats. Exogenous continuous low dose IMD infusion in SHRs resulted in a decrease in $\operatorname{SBP}(24.8 \%, P<0.01)$, DBP (24.1\%, $P<0.01), \mathrm{LV}+\mathrm{dp} / \mathrm{dt}_{\max }(18.0 \%, P<0.01)$, $-\mathrm{LV} \mathrm{dp} / \mathrm{dt}_{\max }(27.0 \%, P<0.01), \operatorname{LVSP}(22.0 \%, P<0.01)$, HR $(12.6 \%, P<0.05)$ and HW/BW $(7.0 \%, P<0.05)$. Decreases in $\operatorname{SBP}(22.0 \%, P<0.01)$, DBP $(24.0 \%, P<0.01)$, $+\mathrm{LV} \mathrm{dp} / \mathrm{dt}_{\max }(25.8 \%, P<0.01),-\mathrm{LV} \mathrm{dp} / \mathrm{dt}_{\max }(37.1 \%, P<0.01)$ $\operatorname{LVSP}(27.3 \%, P<0.01)$, HR $(10.0 \%, P<0.05)$ and HW/BW $(6.7 \%, P<0.05)$ were also observed after ADM administration. The magnitude of the decrease was greater with both $\operatorname{SBP}(12.6 \%, P<0.01)$ and $\operatorname{DBP}(14.1 \%, P<0.05)$ in the ADM-treated SHRs than in the IMD-treated SHRs (Table 2).

Table 2. Effects of IMD on hemodynamic parameters and cardiac function in rats in vivo $(n=11)$.

\begin{tabular}{|c|c|c|c|c|}
\hline & WKY & SHR & SHR+ADM & SHR+IMD \\
\hline $\mathrm{SBP}(\mathrm{mmHg})$ & $124.2 \pm 11.79$ & $240.40 \pm 17.96^{*}$ & $187.50 \pm 24.74^{\dagger}$ & $180.7 \pm 43.5^{\dagger}$ \\
\hline $\mathrm{DBP}(\mathrm{mmHg})$ & $90.11 \pm 18.43$ & $179.90 \pm 19.60^{*}$ & $142.00 \pm 23.72^{\dagger}$ & $136.5 \pm 24.3^{\dagger}$ \\
\hline HR (beats/min) & $351 \pm 11.40$ & $403.80 \pm 22.19^{\#}$ & $363.30 \pm 28.99^{\S}$ & $352.80 \pm 43.63^{\S}$ \\
\hline$+\mathrm{LV} \mathrm{dp} / \mathrm{dt}_{\max }(\mathrm{mmHg} / \mathrm{s})$ & $7735 \pm 1378$ & $10440 \pm 1519^{*}$ & $7746 \pm 1004^{\dagger}$ & $8611 \pm 1314^{\dagger}$ \\
\hline$-\mathrm{LV} \mathrm{dp} / \mathrm{dt}_{\max }(\mathrm{mm} \mathrm{Hg} / \mathrm{s})$ & $5420 \pm 1117$ & $8885 \pm 604.4^{*}$ & $5585.0 \pm 967.9^{\dagger}$ & $6484 \pm 1036^{\dagger}$ \\
\hline LVSP (mmHg) & $143 \pm 9.83$ & $236.30 \pm 17.99^{*}$ & $171.80 \pm 39.41^{\dagger}$ & $184.10 \pm 42.12^{\dagger}$ \\
\hline $\operatorname{LVDP}(\mathrm{mmHg})$ & $-6.32 \pm 2.20$ & $-0.69 \pm 1.40^{\#}$ & $-0.73 \pm 0.55$ & $-0.61 \pm 0.68$ \\
\hline HW/BW (mg/g) & $2.48 \pm 0.08$ & $3.30 \pm 0.05^{*}$ & $3.08 \pm 0.04^{\S}$ & $3.07 \pm 0.08^{\S}$ \\
\hline $\mathrm{BW}(\mathrm{g})$ & $340.1 \pm 10.4$ & $278.9 \pm 4.86^{*}$ & $275.1 \pm 4.6$ & $276.6 \pm 4.13$ \\
\hline
\end{tabular}

Data are expressed as means $\pm \mathrm{SE}, n=8$ in each group. ${ }^{*} P<0.01 ;{ }^{\#} P<0.05$ compared with the WKY group; ${ }^{\dagger} P<0.01 ;{ }^{\S} P<0.05$ compared with the SHR group. IMD: Intermedin; SBP: systolic blood pressure; DBP: diastolic blood pressure; HR: heart rate; LVSP: left ventricular systolic pressure; \pm LV dp/dt max the maximal rates of increase and decrease of left-ventricle pressure development; HW: heart weight; BW: body weight. 


\subsection{Effect of IMD on the expression of CRLR/RAMPs complex in SHRs}

The gene and protein expression levels of CRLR and RAMPs in SHR myocardium and aorta were both significantly higher than those in the WKY rats.

The CRLR mRNA level in the myocardium of the IMD group increased $62.5 \%(P<0.01)$ relative to the SHR control group, and the RAMP mRNA levels of the IMD group decreased $20.3 \%(P<0.05)$ for RAMP1, 48.1\% $(P<0.01)$ for RAMP2 and $44.3 \%(P<0.05)$ for RAMP3 (Figure 1A D) compared to the SHR group. The protein expression levels in the myocardium of the IMD group decreased $62.4 \%(P<0.05)$ for RAMP1 and $59.1 \%(P<0.05)$ for RAMP2 compared to the SHR group, while the CRLR expression increased $52.0 \%$ $(P<0.05)$. The protein expression level of RAMP2 in the ADM group decreased $40.7 \%(P<0.05)$ (Figure $1 \mathrm{E} \sim \mathrm{H})$.
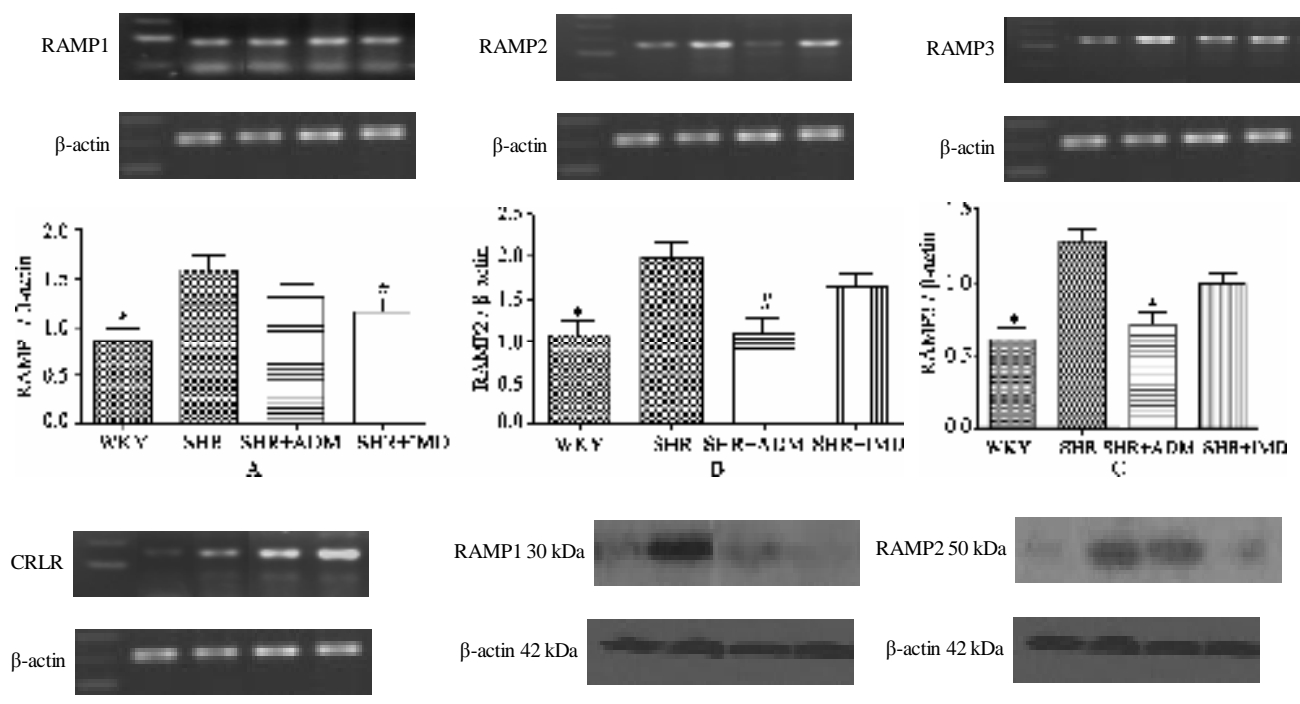

RAMP2 $50 \mathrm{kDa}$

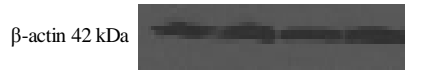

$\beta$-actin $42 \mathrm{kDa}$
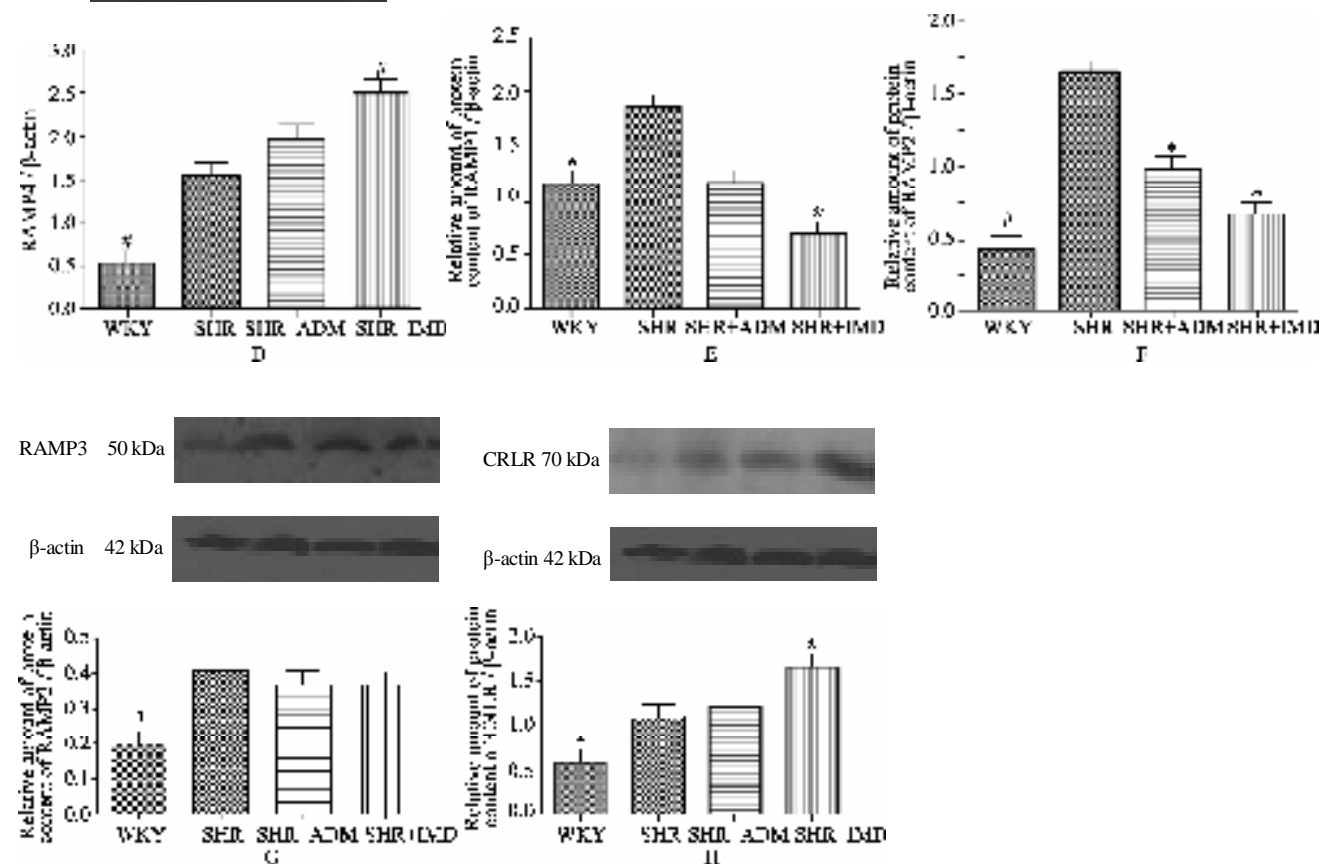

Figure 1. Gene and protein expression levels of RAMP1 (A, E), RAMP2 (B, F), RAMP3 (C, G) and CRLR (D, H) in the myocardium of WKYs, SHRs, adrenomedullin-treated SHRs (SHR+ADM) and intermedin1-47-treated SHRs (SHR+IMD 1-47). Data are expressed as means \pm SE, ${ }^{*} P<0.05$ and ${ }^{\#} P<0.01$ vs. SHR. RAMPs: receptor activity-modifying proteins; CRLR: calcitonin receptor-like receptor; WKYs: Wistar Kyoto (WKY) rats group; SHRs: spontaneously hypertensive rats group. $n=11$ in each group. 
In the aorta, the mRNA contents of the IMD group decreased 24\% $(P<0.05)$ for RAMP1; 72.2\% $(P<0.01)$ for RAMP2; $25.7 \%(P<0.05)$ for RAMP3 and 30.4\% $(P<0.05)$ for CRLR compared to the SHR group. In the ADM group, mRNA levels decreased 50.3\% $(P<0.01)$ for RAMP1; 89.7\% $(P<0.01)$ for RMAP2; 58.0\% $(P<0.01)$ for RMAP3 and $57.5 \%(P<0.01)$ for CRLR compared to the SHR group. Compared to the ADM group, the IMD group contained higher mRNA levels for RAMP1 53.6\% $(P<0.05)$;
RAMP2 $170.4 \%(P<0.05)$; RAMP3 $77.2 \%(P<0.05)$ and CRLR $64.0 \%(P<0.05)$, respectively (Figure $2 \mathrm{~A} \sim \mathrm{D})$. The aortic protein expression level of the IMD group decreased $33.8 \%(P<0.05)$ for CRLR; $23.1 \%(P<0.05)$ for RAMP1; $20.9 \%(P<0.05)$ for RAMP2 and $22.8 \%(P<0.01)$ for RAMP3 relative to the SHR group. The RAMP1 expression level in the ADM group decreased 19.3\% $(P<0.05)$, and the RAMP2 expression level decreased $12.7 \%(P<0.05)$ compared to the SHR group (Figure $2 \mathrm{E} \sim \mathrm{H}$ ).
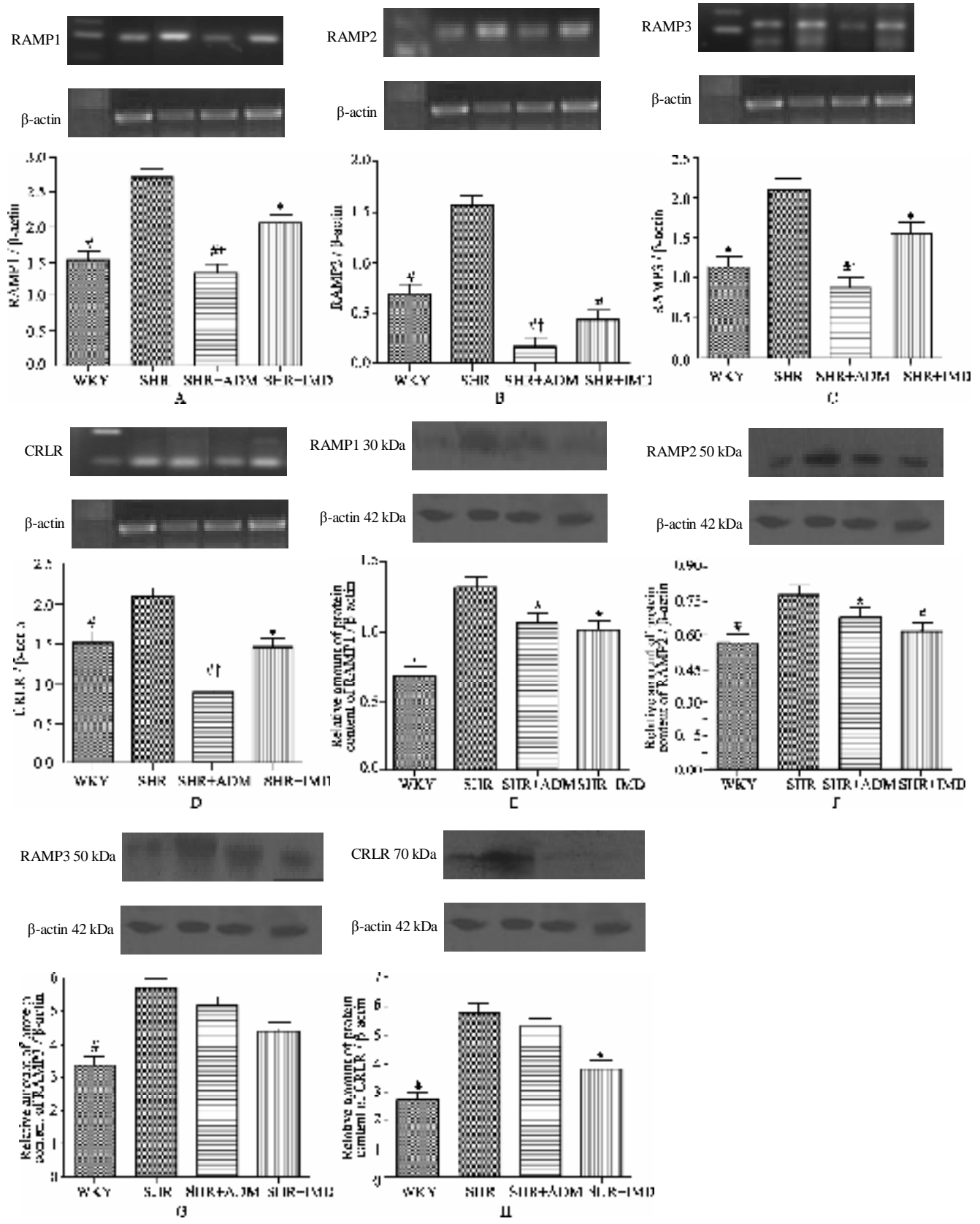

Figure 2. Gene and protein expression levels of RAMP1 (A, E), RAMP2 (B, F), RAMP3 (C, G) and CRLR (D, H) in the aorta of WKYs, SHRs, adrenomedullin-treated SHRs (SHR+ADM) and intermedin 1-47-treated SHRs (SHR+IMD 1-47). Data are expressed as means \pm SE, ${ }^{*} P<0.05$ and ${ }^{\#} P<0.01$, vs. SHR, ${ }^{\dagger} P<0.05$ vs. SHR + IMD. RAMPs: receptor activity-modifying proteins; CRLR: calcitonin receptor-like receptor; WKYs: Wistar Kyoto (WKY) rats group; SHRs: spontaneously hypertensive rats group. $n=11$ in each group. 


\subsection{The gene expression levels of ANP and BNP in myocardium}

The myocardial mRNA levels of the SHR group were $105.1 \%(P<0.05)$ higher for ANP and $57.3 \%(P<0.05)$ higher for BNP than the corresponding values in the WKY group. The ANP mRNA level in the IMD group was $87.4 \%$ $(P<0.01)$ higher than in the SHR group and 66.4\% $(P<0.01)$ higher than the ANP concentration in the ADM group. The BNP mRNA level in the IMD group was $58.1 \%(P<0.01)$ higher than in the SHR group and $74.0 \%(P<0.01)$ higher than the BNP concentration in the ADM group (Figure 3).

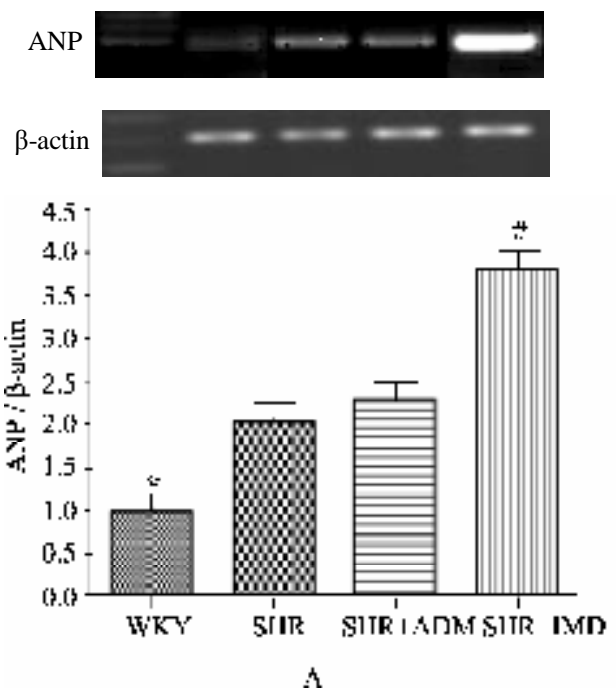

\subsection{The effect of IMD on cAMP production in SHRs}

The myocardial cAMP content of the SHR group was $19.4 \%(P<0.05)$ lower than in the WKY group. The IMD group contained $30.2 \%(P<0.05)$ less cAMP than the SHR group (Figure 4 ). In the aorta, the cAMP content was $44.4 \%$ $(P<0.05)$ higher in the SHR group than in the WKY group. The aortic cAMP level was markedly enhanced in the IMD group, with a $53.0 \%(P<0.01)$ increment compared to the SHR group, and a $43.0 \%(P<0.01)$ increase relative to the ADM group (Figure 4).
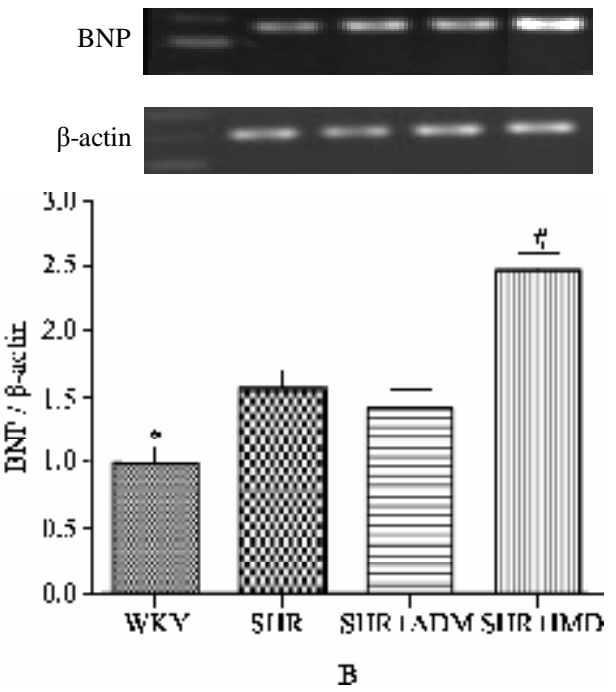

Figure 3. Gene expression levels of ANP (A) and BNP (B) in the myocardium of WKYs, SHRs, adrenomedullin-treated SHRs (SHR+ADM) and intermedin-treated SHRs (SHR+IMD). ANP and BNP mRNA levels are normalized to that of $\beta$-actin for semi-quantitative analysis. Data are expressed as means $\pm \mathrm{SE},{ }^{*} P<0.05 v$ s. SHR, ${ }^{\#} P<0.01$ vs. SHR and SHR+ADM. ANP: atrial natriuretic peptide; BNP: brain natriuretic peptide; WKYs: Wistar Kyoto (WKY) rats group; SHRs: spontaneously hypertensive rats group. $n=11$ in each group.

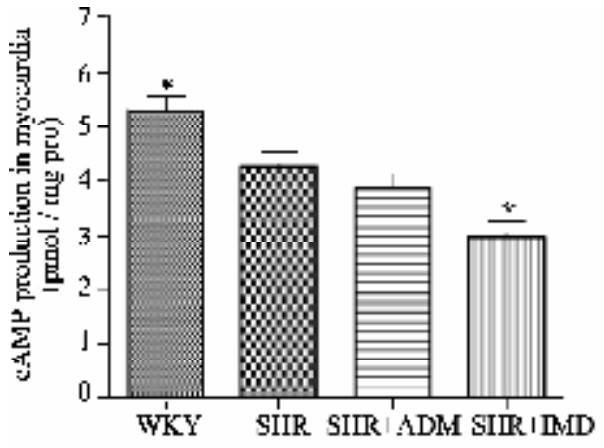

A

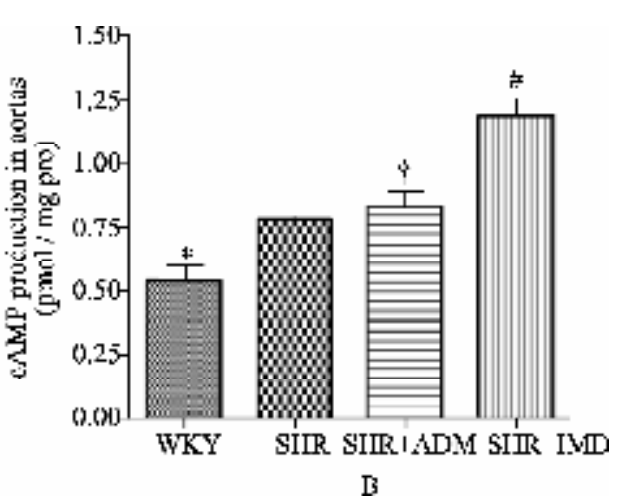

I

Figure 4. Effect of IMD on the cAMP content in SHR myocardium (A) and aorta (B) $(n=8)$. Data are expressed as mean \pm SE, ${ }^{*} P<0.05$ compared with SHR (A), ${ }^{*} P<0.05,{ }^{\#} P<0.01$ compared with SHR, ${ }^{\dagger} P<0.01$ compared with SHR+IMD. IMD: intermedin; SHRs: spontaneously hypertensive rats group. $n=11$ in each group. 


\section{Discussion}

Our study utilized mini-osmotic pumps for continuous administration of IMD and ADM in SHRs. The results demonstrated that low doses of IMD and ADM decreased blood pressure, $\mathrm{LV} \pm \mathrm{dp} / \mathrm{dt}$, LVSP and HR. Our findings showed IMD evokes a stronger hypotensive response compared to ADM, whereas IMD exhibited a weaker effect on $\mathrm{LV} \pm \mathrm{dp} / \mathrm{dt}$ and LVSP than ADM. The differences between them were not statistically significant. This was possibly caused by the use of different animal models, drug regimens, and inadequate number of samples of rats. Furthermore, previous studies were performed on healthy rats with intravenous administration, ${ }^{[6,12,13]}$ whereas our study was carried out on hypertensive rats. Hypertension induces nervous system malfunctions, body fluid imbalance, and blood vessels abnormalities, all of which may influence the IMD response. Secondly, our study employed an alternative drug delivery route at a different administration rate and concentration. The application of mini-osmotic pump for a low-dose $\left(500 \mathrm{ng} / \mathrm{kg}\right.$ per hour), ${ }^{[14,15]}$ slow-release regimen distinguished our approach from the previously performed intravenous injection of high-dose, quick-release formulations. Additionally, it has been demonstrated that when administered intravenously, IMD elicits a hypotensive effect that is weaker than that evoked by ADM at low doses, ${ }^{[12]}$ but induces a stronger response at higher doses. ${ }^{\left[{ }^{6,13]}\right.}$ The observed HR decrease by exogenously administered IMD and ADM is unprecedented, as previous studies have shown an increase in HR. Besides, IMD 1-47 and ADM cause a similar magnitude of decrease in the HW/BW ratio of SHRs. Therefore, as a novel member of the CGRP family, IMD probably confers modulatory effects on the cardiovascular system similar to, or even stronger than, those elicited by ADM and CGRP, which include lowering blood pressure and improving cardiac function. Its actions are associated with elevations of nitric oxide (NO) ${ }^{[16]}$ and $\mathrm{cAMP}^{[17]}$ and possibly synergize the actions of two other cardiovascular peptides, ANP and BNP.

Our results showed up-regulated gene expression of ANP and BNP in the SHR myocardium, which indicates that ANP and BNP alleviate pressure load by reducing cardiac preload and afterload via natriuresis, diuresis and vasodilation ${ }^{[18]}$ as a compensatory response against pressure overload. The myocardial ANP and BNP mRNA expression in SHRs was significantly elevated by exogenous administration of IMD, to an extent greater than that induced by ADM. IMD has demonstrated the ability to evoke antinatriuretic and antidiuretic responses, ${ }^{[7]}$ which is contradictory to the natriuretic and diuretic effects elicited by ANP and BNP. ${ }^{[19]}$
Therefore, this antagonism might induce the increased release of ANP and BNP as a possible secondary response to IMD's anti-natriuretic/diuretic effect. Furthermore, the up-regulation of ANP and BNP gene expression also synergizes the cardioprotective responses to IMD by facilitating its biological effects in reducing volume overload and peripheral blood pressure and improving cardiac function. In vivo, this fully embodies the network-regulatory function between cardiovascular active factors, and this intermolecular regulatory function plays an important role in maintaining normal physiological functions and homeostasis.

Calcitonin/CGRP family members are low-molecularform peptides. They bind to a common receptor system, the CRLR/RAMP system, in addition to their specific receptors. ${ }^{[20]}$ Exogenous administration of IMD 1-47 in SHRs evoked different responses in the gene and protein expression of CRLR and RAMPs in the myocardium. For example, myocardial CRLR was markedly elevated at both the gene and protein expression levels, while myocardials, RAMP1 and RAMP2, were down-regulated at both gene and protein levels. Furthermore, a decreased tendency was observed for both gene and protein expressions of myocardial RAMP3, although this was not statistically significant. The expression inconsistencies among the IMD receptors may be consequences of positive and negative feedback regulations of signal transduction. The up-regulation of CRLR is a possible indication that exogenous IMD modulates the myocardial signal transduction pathways and the activities of associated synthases in SHRs. Moreover, RAMP1 and RAMP2 may act in combination with an alternative receptor or in a receptor-independent manner. Similar inconsistencies have also been reported elsewhere, ${ }^{[21,22]}$ and further investigations are still required to decipher the related mechanisms. Exogenously administered ADM down-regulated myocardial RAMP2 and RAMP3 with no appreciable influence on the RAMP1 level, which is different than the responses evoked by IMD. Therefore, the effects of IMD on cardiac function amelioration and myocardial hypertrophy reduction under hypertensive conditions are possibly exerted through the RAMP1/CRLR and RAMP2/CRLR receptors. In the aorta, exogenous IMD infusion significantly down-regulated the expression of the CRLR/RAMPs receptor complexes, indicating the vasodilator and hypotensive effects of IMD are elicited nonspecifically via RAMPs (CRLR/RAMP1-3). ${ }^{[17]}$

Our previous studies have already demonstrated that IMD 1-47, IMD 8-47 and IMD 1-53 can stimulate cAMP production both in vivo and in isolated rat myocardium and aorta. ${ }^{[5,23]}$ In this study, the myocardial cAMP concentration in SHRs was diminished after exogenous administration of 
IMD 1-47 but was unaffected by ADM infusion. It is possible that elevation of the cardiac sympathetic nervous activity during heart failure reduces the density of betaadrenergic receptors and, therefore, the concentration of cAMP $^{[24-26]}$. Additionally, the increased myocardial ANP and BNP gene levels in SHRs, especially after administration of IMD 1-47, may also have inhibited myocardial cAMP production. ${ }^{[27,28]}$ In contrast, the arterial cAMP concentration in SHRs was markedly increased after exogenous administration of IMD 1-47. We thus postulate that IMD exerts its biological function via an increase of the intracellular cAMP level by non-selectively activating the CRLR/ RAMPs receptor complexes. ${ }^{[17]}$ This may have stimulated the tremendous cAMP release in aortas, serving to relax blood vessels and reduce blood pressure.

The mechanism of the IMD-induced hypotensive effect has not been completely elucidated. The vasodilator effect of IMD is known to be associated with elevation of aortic NO production, ${ }^{[16,29]}$ suppression of angiotensin II-induced vascular smooth muscle cell proliferation (to be presented in another publication) and increases of cellular ${ }^{[1]}$ and aortic ${ }^{[23]}$ cAMP concentrations. Recently, considerable attention has been paid to experiments involving IMD and its receptors. In the rat left coronary ligation model of ischemic heart failure, elevated RAMP2 mRNA expression levels are observed in both ischemic and non-ischemic left ventricles, although larger changes are seen in the ischemic left ventricle. ${ }^{[30]}$ In the nitric oxide synthase inhibitor-induced pressure overload rat model, the expression of IMD, ADM and their receptors in left ventricular cardiomyocytes are substantially up-regulated. In these cells, ADM, CRLR, RAMP2, and RAMP3 can be restored to normal abundance after hydralazine/hydrochlorothiazide treatment, which results in normalization of SBP and hypertrophic marker gene expression. In contrast, the IMD and RAMP1 levels remain elevated and the myocardial thickness does not recover after such treatments. This demonstrates the crucial role of IMD in the pathology of hypertensive myocardial hypertrophy in this animal hypertension model. ${ }^{[16]}$ In the rat model of isolated lung perfusion, IMD reduces pulmonary artery pressure. Furthermore, its hypotensive effect is found to be significantly attenuated by CGRP 8-37 and $L-\mathrm{NAME}$ $\left(N^{\mathrm{w}}\right.$-nitro- $L$-arginine methyl ester), indicating that IMD produces pulmonary vascular dilation through the CGRP1 receptor and NO pathway. ${ }^{[31]}$ Correspondingly, in the spontaneously hypertensive stroke-prone rats, the expression of ADM, RAMP2 and CRLR receptors are elevated. ${ }^{[32]}$ During pregnancy, the concentrations of all three RAMPs and CRLR receptors increase in a progesterone-dependent manner. ${ }^{[33,34]}$ In the renal fibrosis model, the expressions of
RAMP1 and RAMP2 are raised to varying degrees. ${ }^{[35]}$ In the sepsis model, the RAMP3 level is significantly elevated, implying that RAMP3 may interact with receptors other than CRLR, or may act in a receptor-independent manner. ${ }^{[21]}$

This study shows that exogenous administration of IMD 1-47 using mini-osmotic pumps prominently reduces arterial blood pressure and improves cardiac function in SHRs. Down-regulation in the high-level expression of the genes and proteins in the aortic IMD receptor system, such as RAMPs/CRLR, and high-level expression of myocardial RAMP1 and RAMP2 proteins were also observed in SHRs. Furthermore, exogenous IMD 1-47 in SHRs increases the aortic cAMP concentration, but reduced the myocardial cAMP content. Therefore, we infer that exogenous administration of IMD 1-47 can stimulate aortic cAMP production and high-level myocardial ANP and BNP expression in SHRs via decreasing the IMD receptor complex abundance to ultimately reduce SHR peripheral blood pressure and ameliorate cardiac function. These results suggest IMD might regulate cardiovascular function and myocardial hypertrophy as a cardiovascular active peptide and exert its biological function through cAMP, ANP and BNP modulation. It is worth investigating further whether the IMD receptor could be a new target in preventing and treating chronic hypertension.

\section{Acknowledgments}

This work was supported by the National Natural Sciences Foundation of China (Grant No. 30570738), the Program for New Century Excellent Talents in University of the Education Ministry of China (NCET-05-0016). The authors declare that they have no competing interests.

\section{References}

1 Roh J, Chang CL, Bhalla A, et al. Intermedin is a calcitonin/calcitonin gene-related peptide family peptide acting through the calcitonin receptor-like receptor/receptor activity-modifying protein receptor complexes. J Biol Chem 2004; 279: 7264-7274.

2 Wimalawansa SJ. Amylin, calcitonin gene-related peptide, calcitonin, and adrenomedullin: a peptide superfamily. Crit Rev Neurobiol 1997; 11: 167-239.

3 Takei Y, Inoue K, Ogoshi M, et al. Identification of novel adrenomedullin in mammals: a potent cardiovascular and renal regulator. FEBS Lett 2004; 556: 53-58.

4 Poyner DR, Sexton PM, Marshall I, et al. The mammalian calcitonin gene-related peptides, adrenomedullin, amylin, and calcitonin receptors. Pharmacol Rev 2002; 54: 233-246. 
5 Yang JH, Jia YX, Pan CS, et al. Effects of intermedin (1-53) on cardiac function and ischemia/reperfusion injury in isolated rat hearts. Biochem Biophys Res Commun 2005; 327: 713-719.

6 Fujisawa Y, Nagai Y, Miyatake A, et al. Roles of adrenomedullin 2 in regulating the cardiovascular and sympathetic nervous systems in conscious rats. Am J Physiol Heart Circ Physiol 2006; 290: H1120-H1127.

7 Fujisawa Y, Nagai Y, Miyatake A, et al. Renal effects of a new member of adrenomedullin family, adrenomedullin2, in rats. Eur J Pharmacol 2004; 497: 75-80.

8 Saita M, Shimokawa A, Kunitake T, et al. Central actions of adrenomedullin on cardiovascular parameters and sympathetic outflow in conscious rats. Am J Physiol 1998; 274: R979-R984.

9 Takahashi H, Watanabe TX, Nishimura M, et al. Centrally induced vasopressor and sympathetic responses to a novel endogenous peptide, adrenomedullin, in anesthetized rats. Am J Hypertens 1994; 7: 478-482.

10 Zeng Q, Yuan Y, Wang X, et al. Upregulated expression of intermedin and its receptor in the myocardium and aorta in spontaneously hypertensive rats. Peptides 2009; 30: 391-399.

11 Fujisawa Y, Nagai Y, Miyatake A, et al. Effects of adrenomedullin 2 on regional hemodynamics in conscious rats. Eur J Pharmacol 2007; 558: 128-132.

12 Taylor MM, Bagley SL, Samson WK. Intermedin/adrenomedullin-2 acts within central nervous system to elevate blood pressure and inhibit food and water intake. Am J Physiol Regul Integr Comp Physiol 2005; 288: R919-R927.

13 Ren YS, Yang JH, Zhang J, et al. Intermedin 1-53 in central nervous system elevates arterial blood pressure in rats. Peptides 2006; 27: 74-79.

14 Nishikimi T, Yoshihara F, Horinaka S, et al. Chronic administration of adrenomedullin attenuates transition from left ventricular hypertrophy to heart failure in rats. Hypertension 2003; 42: 1034-1041.

15 Nishikimi T, Mori Y, Kobayashi N, et al. Effect of chronic adrenomedullin infusion in Dahl salt-sensitive rats. Hypertension 2002; 39: 1077-1082.

16 Zhao Y, Bell D, Smith LR, et al. Differential expression of components of the cardiomyocyte adrenomedullin/intermedin receptor system following blood pressure reduction in nitric oxide-deficient hypertension. J Pharmacol Exp Ther 2006; 316: 1269-1281.

17 Chang CL, Roh J, Hsu SY. Intermedin, a novel calcitonin family peptide that exists in teleosts as well as in mammals: a comparison with other calcitonin/intermedin family peptides in vertebrates. Peptides 2004; 25: 1633-1642.

18 Nakao K, Ogawa Y, Suga S, et al. Molecular biology and biochemistry of the natriuretic peptide system. II: Natriuretic peptide receptors. J Hypertens 1992; 10: 1111-1114.

19 Marin-Grez M, Fleming JT, Steinhausen M. Atrial natriuretic peptide causes pre-glomerular vasodilatation and post-glomerular vasoconstriction in rat kidney. Nature 1986; 324: 473-476.

20 Fischer JA, Muff R, Born W. Functional relevance of G-protein-coupled-receptor-associated proteins, exemplified by receptor-activity-modifying proteins (RAMPs). Biochem Soc Trans 2002; 30: 455-460.

21 Ono Y, Okano I, Kojima M, et al. Decreased gene expression of adrenomedullin receptor in mouse lungs during sepsis. Biochem Biophys Res Commun 2000; 271: 197-202.

22 Uzan B, de Vernejoul MC, Cressent M. RAMPs and CRLR expressions in osteoblastic cells after dexamethasone treatment. Biochem Biophys Res Commun 2004; 321: 802-808.

23 Pan CS, Yang JH, Cai DY, et al. Cardiovascular effects of newly discovered peptide intermedin/adrenomedullin 2 . Peptides 2005; 26: 1640-1646.

24 Anderson KM, Eckhart $\mathrm{AD}$, Willette $\mathrm{RN}$, et al. The myocardial beta-adrenergic system in spontaneously hypertensive heart failure (SHHF) rats. Hypertension 1999; 33: 402-407.

25 Brodde $\mathrm{OE}$, Bruck $\mathrm{H}$, Leineweber $\mathrm{K}$, et al. Presence, distribution and physiological function of adrenergic and muscarinic receptor subtypes in the human heart. Basic Res Cardiol 2001; 96: 528-538.

26 Kaye DM, Smirk B, Finch S, et al. Interaction between cardiac sympathetic drive and heart rate in heart failure: modulation by adrenergic receptor genotype. J Am Coll Cardiol 2004; 44: 2008-2015.

27 Kim NN, Huang Y, Moreland RB, et al. Cross-regulation of intracellular cGMP and cAMP in cultured human corpus cavernosum smooth muscle cells. Mol Cell Biol Res Commun 2000; 4: 10-14.

28 McGrath MF, de Bold ML, de Bold AJ. The endocrine function of the heart. Trends Endocrinol Metab 2005; 16: 469-477.

29 Yang JH, Pan CS, Jia YX, et al. Intermedin1-53 activates L-arginine/nitric oxide synthase/nitric oxide pathway in rat aortas. Biochem Biophys Res Commun 2006; 341: 567-572.

30 Oie E, Vinge LE, Yndestad A, et al. Induction of a myocardial adrenomedullin signaling system during ischemic heart failure in rats. Circulation 2000; 101: 415-422.

31 Burak Kandilci H, Gumusel B, Wasserman A, et al. Intermedin/ adrenomedullin-2 dilates the rat pulmonary vascular bed: dependence on CGRP receptors and nitric oxide release. Peptides 2006; 27: 1390-1396.

32 Wang X, Nishikimi T, Akimoto K, et al. Upregulation of ligand, receptor system, and amidating activity of adrenomedullin in left ventricular hypertrophy of severely hypertensive rats: effects of angiotensin-converting enzyme inhibitors and diuretic. J Hypertens 2003; 21: 1171-1181.

33 Dong YL, Vegiraju S, Chauhan M, et al. Expression of calcitonin gene-related peptide receptor components, calcitonin 
receptor-like receptor and receptor activity modifying protein 1 , in the rat placenta during pregnancy and their cellular localization. Mol Hum Reprod 2003; 9: 481-490.

34 Thota C, Gangula PR, Dong YL, et al. Changes in the expression of calcitonin receptor-like receptor, receptor activitymodifying protein (RAMP) 1, RAMP 2, and RAMP 3 in rat uterus during pregnancy, labor, and by steroid hormone treatments. Biol Reprod 2003; 69: 1432-1437.

35 Nagae T, Mukoyama M, Sugawara A, et al. Rat receptoractivity-modifying proteins (RAMPs) for adrenomedullin/ CGRP receptor: cloning and upregulation in obstructive nephropathy. Biochem Biophys Res Commun 2000; 270: 89-93. 